\title{
Relationship between Creative Inspiration, Self-Evaluated Satisfaction and Brain Activity through Simplified Music Composition
}

\author{
Hiromu Sato $^{\mathrm{a}}$, Yuya Chiba ${ }^{\mathrm{b}}$, Kenji Moriya ${ }^{\mathrm{a},{ }^{*}}$, Masahiro Nakagawa ${ }^{\mathrm{c}}$ \\ aDepartment of Production Systems Engineering, National Institute of Technology, Hakodate college, \\ 14-1 Tokura, Hakodate City, Hokkaido, 042-8501, Japan

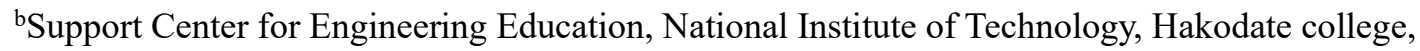 \\ 14-1 Tokura, Hakodate City, Hokkaido, 042-8501, Japan \\ 'Department of Science of Technology Innovation, Nagaoka University of Technology, \\ 1603-1 Kamitomioka, Nagaoka City, Niigata, 940-2188, Japan \\ *Corresponding Author: moriya@hakodate-ct.ac.jp
}

\begin{abstract}
The human prefrontal cortex (PFC) is an important target for a research which attempt to elucidate and control at will of higher brain function using non-invasive neuroimaging techniques. In particular, creativity is specific higher human brain function and integral for evolution of human society. Creative inspirations generated during creative activity often solve hard problems and lead us in the good direction. The present study examined the PFC activity when creative inspiration happened and an influence of difference of brain activity on quality of creation, using the simplified music composition task and near infrared spectroscopy (NIRS). Deeply understanding these brain function possibly builds a foundation for development of methods to stimulate brain activation for creativity.

The PFC activity measurements were achieved by measuring oxygen metabolism in cerebral blood flow (CBF) using 10-channel wearable optical topography (WOT-100, $\mathrm{NeU}$ Ltd.). We invested new simplified method of music composition to analyze brain activity of a person without instruments experience. A report of creative inspirations was conducted by raising subject's hand as possible as small during the music composition task and we compared statistically brain activity of before and after inspirations. Correlation coefficient between brain activity and selfevaluation measured by visual analog scale (VAS) was investigated.

The present research found that right ventrolateral PFC is activated by creative inspiration. Significant brain
\end{abstract}

activation of 4 subjects out of participated 5 subjects was observed $(p<0.05)$. The CBF increases delayed from inspirations and mean delay time was approximately 12 seconds. We observed weak positive correlation between brain activity and self-evaluation in a wide range. However, correlation coefficient was not statistically significant $(p>$ 0.05). Simplification of music composition task possibly mask clear correlation. In conclusion, the main finding that right PFC activation by inspirations indicated important mechanism of creativity.

Keywords: NIRS, Creativity, Inspiration, Music composition.

\section{Introduction}

To bring out and control freely our brain ability that has high potential are desirable goal for more improvement of our prosperous lifestyle. Nevertheless, the human brain mechanism, especially the higher brain functions such as creativity, complex emotions and thought are not elucidated significantly even today. The elucidation of these mechanism is helpful and important for improvement and application of various field such as medical, commercial and so on. The prefrontal region of human brain is focused in the field of higher brain functional research since this region is developing specifically in comparison to other animals. In order to elucidate these, various researches have examined emotion estimation using brain activity significantly in a wide range from children to adults and one of the important factor to elucidation of the higher brain functions ${ }^{(1-3)}$. 
On the other hand, creativity is also important brain function extremely for improvement of our technics, culture, namely prosperous lifestyle. Actually, creative activities and/or creations such as music are also important factors in healing and relaxation during daily life ${ }^{(4)}$. When we tackle these creative activities, we often receive creative inspiration that is coming to mind suddenly good ideas. Then people obtain high-satisfaction since this inspiration sometimes creates outstanding objects, solve serious problem and lead circumstances in a good direction. Previous inspiration study have demonstrated that the cerebral blood flow (CBF) in the prefrontal cortex (PFC) increase by inspiration that is obtained using detective task of morphing image change ${ }^{(5)}$. In addition, Previous study which use creative tasks indicated that bilateral frontal activity in musicians greater than non-musicians during creative task and creativity is supported by increased frontal cortical activity ${ }^{(6)}$. However, researches which focus on creative inspiration and has attempt to reveal relationship between creative inspiration and brain activity are not adequate ${ }^{(7)}$. Creativity is based on divergent thinking that search ideas in various field without restrict of boundary and create enough answers including flexible ideas to open-ended problems ${ }^{(8)}$. Therefore, we guess that using a music is suitable for examine creative inspiration since, in general, ease to search ideas and receive inspirations because a music is extremely pervasive creation and abundant experiences and knowledge are accumulated inside of people. The present research focused on creative activity, in which the formation of a good ideas and the enthusiasm namely, creative inspiration that develops occasionally enables performing high-quality work in a project. We adopted music composition as creative task and invested new simplified music composition method that everyone can create a music since the people who have never experienced instruments cannot compose a music generally.

Neuroimaging techniques such as electroencephalogram (EEG) and functional magnetic resonance imaging (fMRI) is frequently used for measurement of human brain activity since both methods are non-invasively. Similarly, in recent years, neuroscientists also frequently use a CBF change of the frontal cortex determined using near infrared spectroscopy (NIRS) that is non-invasive as index of brain activity, despite having several drawbacks in comparison to other neuroimaging techniques. For instance, NIRS can only obtain information of brain's surface layer and has poor spatial resolution. Nevertheless, NIRS present several distinguished advantages compared with other measuring methods: simplicity of use and administration; less physical and psychological burden than existing neuroimaging techniques since low restriction of body movement; high robust with extrinsic noise in the environment such as electromagnetic wave $^{(9)}$. Thus, NIRS can measure brain activity in state that is similar ordinary life and suitable for experiment using sound element such as music because huge noise is not occurred like fMRI.

The PFC has the higher brain functions such as creativity. Additionally, the decision making not logically, but intuitively is called the heuristic decision making and reported that associate with activation of orbitofrontal cortex $(\mathrm{OFC})^{(10)}$. Thus, we speculated that creativity or creative inspiration related to OFC activity and hypothesized that the ventral PFC is activated by creative inspiration. We measured characteristics of brain activity change when creative inspiration was reported and investigated the relationship between self-evaluation, and brain activity during a music composition task. Investigating this may provide a foundation for elucidation of relationship between creativity and brain activity, the development of methods to stimulate brain activation for creativity by combining brain stimulation methods which are investigated actively and applied clinically ${ }^{(11,12)}$. We guess that creative inspiration is frequently used and important for not only the person who is creator such as musician but also researcher and developer and integral for development of our technics.

\section{Materials and Methods}

\subsection{Participants}

We examined data from healthy five participants (5 males, age range: 19 21 years) who are belonging to National Institute of Technology, Hakodate college as student. In accordance with the regulation set forth by the Life Ethics Committee of the National Institute of Technology, Hakodate college, we explained details and safety of experiment specifically and informed consent was obtained from all participants. Only one participant out of five participants had experience of playing the keyboard instrument and other participants have not played the instruments. All participants have never composed a music but, listened the music on a daily basis.

\subsection{Creative Task: Simplified Music Composition}

The music composition task involved the composition of instrumental pieces (vocal composition was not included) of a genre that is Rock music chosen by considering amount 
of knowledge and experience of subjects. We created three instruments phrases in common key (A-minor) and tempo $(120 \mathrm{bpm})$ in advance: drum phrases that were prepared 11 phrases each length of 8 bars by programing; accompaniment electric guitar phrases that were prepared 7 kinds of chord progression each length of 8 bars based on diatonic chord by recording the played electric guitar phrases. Moreover, these 7 chord progressions were created 3 sets and labeled "slow", "middle", "fast" by amount of sound per 1bar; melodious electric guitar phrases were prepared 11 phrases each length of 4 bars to create various combinations because we hypothesized that melody is the most important element in music for ordinary people with the same method as accompaniment electric guitar. These phrases were composed the A-minor pentatonic scale (Notes: A, C, D, E, G). Melodious electric guitar phrases were also labeled "slow", "middle", "fast" as similar as accompaniment electric guitar to roughly indicate phrases content to subjects. We asked subject to compose a music by choosing and combining these phrases freely using Digital Audio Workstation (DAW) software on a personal computer without playing the instruments. Whole length of music is 24 bars and separated 3 sections each 8 bars. The first section is created in advance for more simplify. Thus, subjects created 2 sections of the first section continuous. Figure 1 illustrates overview of music composition methods.

\subsection{Creative Inspiration}

In the present study, we defined creative inspiration as two follows: subjects come up with good plan, idea of composition and phrase initiatively; subjects receive some stimulus from prepared musical phrases or created pieces. We explained these definitions to all subjects before the beginning of experiments. Subjects reported inspiration by judging subjective perception following these definitions.

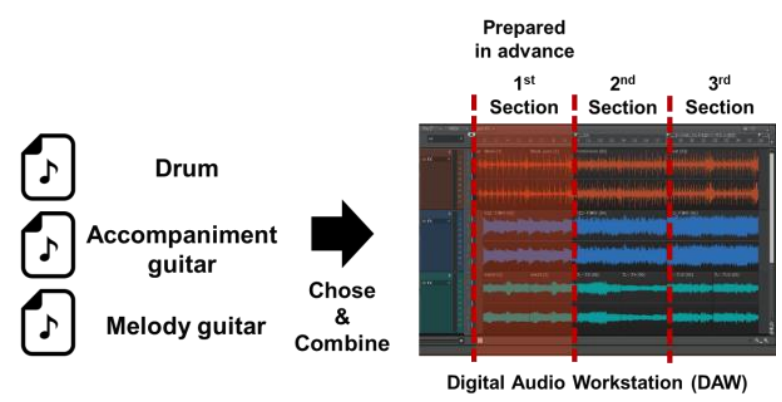

Fig. 1. Illustration of simplified music composition method. Subjects chose prepared phrases and combine on the DAW software. Light fill part on the DAW picture indicates $1^{\text {st }}$ section that was created in advance.

\subsection{Experimental Procedure}

We quantitatively evaluated the brain activity of the subject as they performed a music composition task following a rest task. During the experiment, the subject sat on a chair facing the screen of a personal computer. The subject was instructed to keep their eyes closed during the rest task. The experimental procedure is shown in Figure 2. The first $1 \mathrm{~min}$ of the procedure comprised a set "Rest" period. During the "Listening the piece" period, we asked subjects to listen to the first section piece of music until subjects memorize. After these tasks, subjects proceeded "Composition" task. The cycle of "Composition" and "Listening the piece" was repeated 2 times each 1 experiment since pieces which should be created by subjects were separated 2 sections. In the second "Listening the piece", subjects listened to the first and second section piece of music until subjects memorize. When subjects received an inspiration, we asked subjects to raise their hands as possible as small and we recorded the time. After subjects completed compose two sections, subjects evaluated self-evaluation of created respective piece subjectively using the visual analog scale (VAS). VAS is frequently used methods to assess subjective sensibility and feeling in widely fields including medical field. It consists of a line usually $100 \mathrm{~mm}$ in length, with index description such as "not satisfy" and "satisfy significantly" in both ends of a line. Subjects reflected their perception and marked point on a line. We measured location of points to 1 [mm] accuracy. Thus, this results became 101points scale from 0 to 100 points $^{(13,14)}$.

\subsection{Measurement and Analysis of Brain Activity}

We quantitatively evaluated brain activity in the PFC by measuring changes in the concentration of oxygenated and deoxygenated hemoglobin (oxy-Hb and deoxy-Hb, respectively) using 10-channel wearable optical topography (WOT-100, NeU Ltd.) with NIRS at a sampling frequency of $5 \mathrm{~Hz}$. This method uses near-infrared ray $(700-900 \mathrm{~nm})$ that has high bio-permeability. With the change in the oxidation state of hemoglobin, the intensity of the transmitted nearinfrared ray changes because the absorption spectra of oxy-

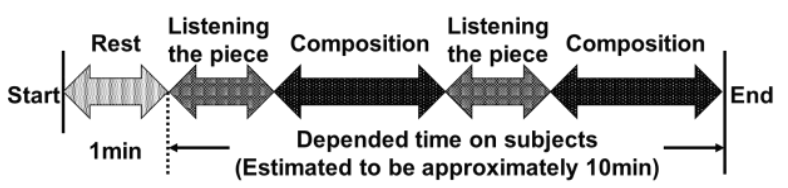

Fig. 2. Timeline illustrating the experimental procedure. 
$\mathrm{Hb}$ and deoxy-Hb are different. Therefore, the change in oxidation state can be evaluated from the intensity change of the ray. These indexes are highly associated with brain functional activation, thus providing reliable indicators of nervous activity for several different tasks. A CBF increases during neural activity are greater than associated activity increases of oxygen metabolism such as oxygen consumption in neuron ${ }^{(15,16)}$. This imbalance between supply and demand that is called neurovascular uncoupling is indicated that oxy-Hb follow a CBF change and decrease in deoxy-Hb as a result of neural activity. Therefore, typically, an increase in oxy-Hb and decrease in deoxy-Hb indicates that the PFC is active compared with the previous condition and a decrease in oxy- $\mathrm{Hb}$ and increase in deoxy- $\mathrm{Hb}$ indicates that the PFC is inactive (Table 1).

We used 15 seconds mean value to analysis since considering time-lag between neuronal response and a CBF change, rising and falling time ${ }^{(17)}$. To eliminate the influence of sudden change, 15 seconds of before switch between task and after one were excluded from analysis. The duration of music composition task was separated into 15-second segments and the mean data was used in analysis. The measurement regions of the PFC corresponding to each measurement channel $(\mathrm{CH})$ are shown in Figure 3 . We investigated the following two factors: to evaluate effect of

Table. 1. Relationship of brain activity with changes in concentrations of oxygenated and deoxygenated hemoglobin.

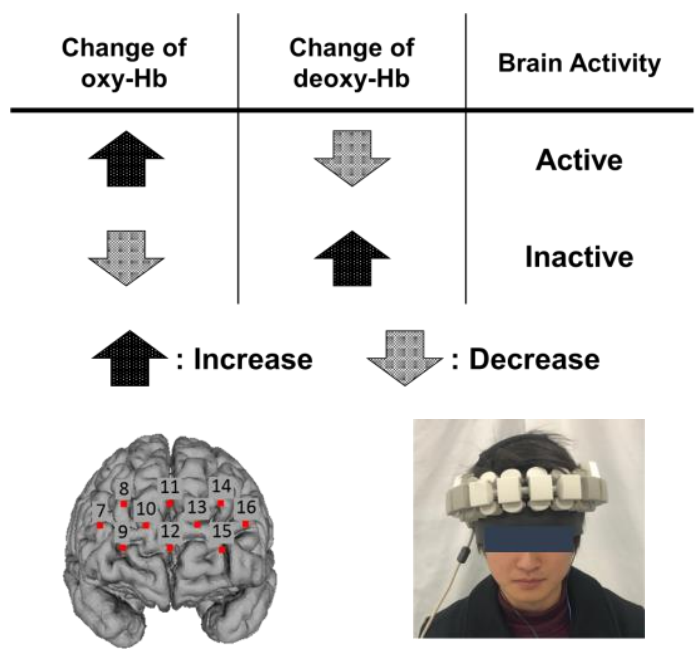

Fig. 3. Regions of the prefrontal cortex (left image) corresponding to the measurement channels of the present study. Measurements of oxygenated and deoxygenated hemoglobin concentrations were conducted using 10-channel wearable optical topography (right image). creative inspiration to brain functional activation, we calculated mean values of oxy- and deoxy-Hb concentration change in 15 seconds after an inspirations were reported and before in 15 seconds and conducted statistical test whether there is significant difference at significance level $5 \%$; to confirm correlation between brain functional activation and subjective self-evaluation, we investigated the relationship between average values of oxy-Hb density change when brain activity was activated and self-evaluation that was measured using VAS. Similarly, correlation coefficient was verified statistically at significance level $5 \%$.

\section{Results and Discussion}

We conducted the experiment each 3 times to all subjects, thus obtained 30 data of brain activity during the music composition task. In total, creative inspirations were reported 15 times: subject A (a person with keyboard instruments experienced) reported 8 times; subject $\mathrm{B}$ reported 1 time; subject $\mathrm{C}$ reported 2 times; subject $\mathrm{D}$ reported 3 times; subject $\mathrm{E}$ reported 1 time. Subject A reported many inspirations compared with other subjects. This amount difference of inspirations implied that difference between a person who have music experience and who did not have.

\subsection{Brain Activity Related to Creative Inspiration}

In several experiments, $\mathrm{A}$ CBF change in all channels had a tendency to increase after creative inspirations were reported. In analysis through all experiments using paired ttest, we observed significance oxy-Hb increases and deoxy$\mathrm{Hb}$ decreases in $9 \mathrm{CH}$ only $(p<0.05)$ namely, right ventrolateral PFC was activated by creative inspiration. A $\mathrm{CBF}$ change was used to demonstrate oxy-Hb. Figure 4 illustrates 30 seconds mean oxy-Hb concentration change in $9 \mathrm{CH}$ after creative inspirations were reported using mean previous 15 seconds value as control. In the Figure4, each oxy- $\mathrm{Hb}$ change of subjects standardized using maximum value of each subjects. We deducted data of Subject $\mathrm{C}$ from this analysis since oxy- deoxy-Hb density change both decreased, we could not judge active or inactive following definition of Table 1. Regarding to a CBF change, subject $\mathrm{A}$ did not have unique reaction in comparison to other subjects unlike the amount of creative inspiration. A speed fluctuation of a CBF had difference every subjects. In particular, a fluctuation of Subject B was fast, on the contrary, fluctuation of Subject D was slow. A peak of every subjects CBF increases delayed from creative inspiration and average was 


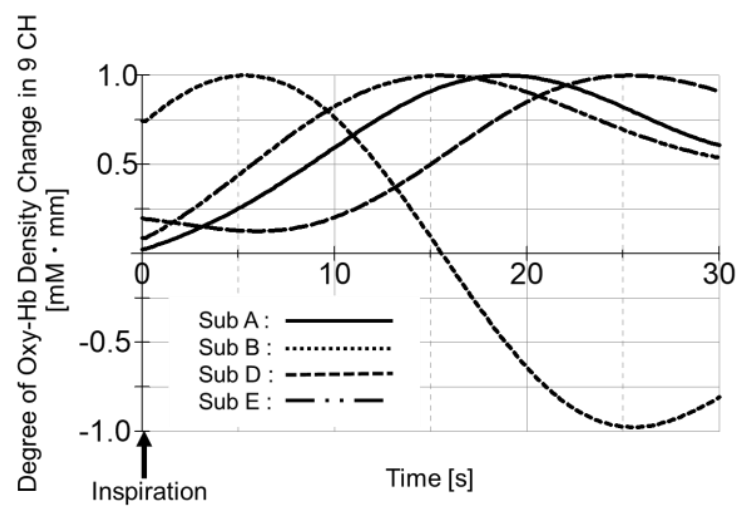

Fig. 4. Mean oxy-Hb concentration change of each subjects in $9 \mathrm{CH}$ for 30 -second after creative inspiration. Point of zero second shows time which were reported creative inspirations.

approximately 12 seconds. Inspiration intensity possibly influenced this difference of fluctuation.

The data from this experiment supported our hypothesis that ventral PFC is activated by creative inspiration. Moreover, several previous research reported that creativity associated with the right PFC and also support our result. A fMRI study of high-level cognitive constructs such as creativity found creativity is related to the ability of right dorsolateral prefrontal cortex and sensorimotor areas ${ }^{(18)}$. During a divergent thinking task that create stories, the right PFC was activated significantly ${ }^{(19)}$. There is a possibility that creative inspiration is trigger these brain functional activation.

\subsection{Relationship between self-evaluation and brain activity}

Mean oxy-Hb concentration change when the PFC activated based on Table 1 during the music composition task was used to demonstrate brain activity using mean oxy-Hb change of rest task as control. Especially, we focused on the medial PFC namely, 11 and $12 \mathrm{CH}$ and analyzed since previous research reported pleasant and unpleasant emotion could asses using the medial PFC activity ${ }^{(20)}$. The correlation analysis between self-evaluation measured by VAS and brain activity indicated a positive correlation. Nevertheless, this correlation coefficient was not statically significant $(p>$ $0.05)$. Figure 5 presents the correlation between selfevaluation and brain activity in $11 \mathrm{CH}$ that were standardized using maximum value of each subjects. Self-evaluation values had a tendency high scores in every subjects $(M=72$, $S D=11, N=30$ ). We considered that this high scoretendency is attributed to simplification of the music

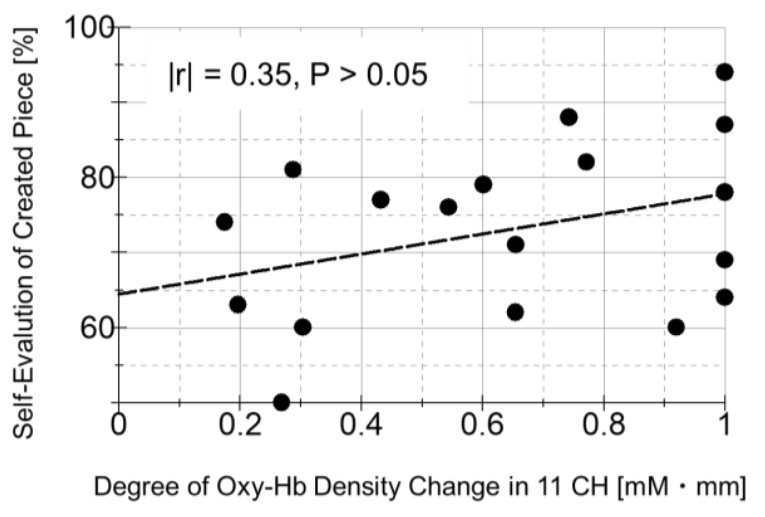

Fig. 5. Correlation between mean oxy-Hb concentration change of all subjects in $11 \mathrm{CH}$ and selfevaluation.

composition task and masked the correlation. Prepared phrases in advance was difficult to deviate from ordinary flow because that was composed based on several combinations of diatonic chord and pentatonic scale of same key. In brief, there was the possibility that all subjects can easily create moderate quality creations.

Elucidation of relationship between self-satisfaction and brain activity is essential for development of brain activation method to propose outstanding creativity. We have to adopt other index of self-satisfaction such as subjective progress or enjoyment instead of self-evaluation.

\section{Conclusions}

In a few limitations that we could not analyze network of each cortex specifically due to difficulty of measure widely cortex area, we examined the brain activity related to creative inspiration and correlation between self-evaluation and brain activity in the PFC. The data of former experiment supported our hypothesis of brain functional activation by creative inspiration. We did not obtain obvious correlation between self-evaluation and brain activity in later experiment. An important finding in the present research was the activation of the $9 \mathrm{CH}$ namely, right ventrolateral PFC by creative inspiration. This result has similarities with previous research, which reported association of creativity and right PFC activation. In addition, intensity of creative inspiration possibly influences a $\mathrm{CBF}$ change since the difference of fluctuation occurred. Therefore, we should measure intensity of inspirations subjectively using VAS in the future work and verified influence.

The difference of inspiration amount between a person with instruments experienced and not one was observed. The 
problems regarding difference of experienced brain activity and relationship between brain activity and amount of inspiration was generated. However, we could not argue statistically because of smallness of sample size in this study. The destination of our research is development of brain activation methods for creativity and providing good quality creative activity for artist, researcher and developer. In order to achieve this destination, we should conduct the further investigation that measure difference of brain activity between person with music composition and instruments experienced and ordinary people and use another indexes such as subjective progress or enjoyment as evaluation of creative activity with increased number of subjects.

\section{Acknowledgment}

The authors thank supporting to a part of this study by a grant from Nagaoka University of Technology for collaborative research with National Institute of Technology 2018, 2019.

\section{References}

(1) Kenji Moriya, Ikusaburo Kurimoto, Nobuo Ezaki and Masahiro Nakagawa : "Influences of Listening to Music in Study Break on Brain Activity and Parasympathetic Nervous System Activity", Journal of the Institute of Industrial Applications Engineers, Vol. 6, No. 1, pp. 3438,2018

(2) Maddalena Mauri, Alessandro Crippa, Andrea Bacchetta, Silvia Grazioli, Eleonora Rosi, Erica Gazzola, Alberto Gallace and Maria Nobile : "The utility of NIRS technology for exploring emotional processing in children”, Journal of Affective Disorders, Vol. 274, No. 6, pp. 819-824, 2020

(3) Hirokazu Doi, Shota Nishitani and Kazuyuki Shinohara : "NIRS as a tool for assaying emotional function in the prefrontal cortex", Frontiers in Human Neuroscience, Vol. 7, No. 770, pp. 1-6, 2013

(4) Yusuke Mitani, Takuya Kubo, Yuya Chiba, Yoshiko Maruyama, Kenji Moriya and Masahiro Nakagawa : "Brain Activity During Listening to And Imagining Music: Does Imagining Music Provide a Similar Effect as Listening to Music?", Journal of the Institute of Industrial Applications Engineers, Vol. 7, No. 4, pp. 127-131, 2019

(5) Keisuke Teranishi and Hiroshi Hagiwara : "Characteristic Changes in the Brain Measured by Near-
Infrared Spectroscopy (NIRS) during "Aha" Experiences", J. Mobile Interactions, Vol. 1, No. 1, pp. 41-46, 2011 (in Japanese)

(6) Crystal Gibson, Bradley S. Folley and Sohee Park : "Enhanced divergent thinking and creativity in musicians: A behavioral and near-infrared spectroscopy study", Brain and Cognition, Vol. 69, N0. 1, pp. 162-169, 2009

(7) Hiromu Sato, Yuya Chiba, Yoshiko Maruyama, Kenji Moriya, and Masahiro Nakagawa : "Investigation of Relationship Between Progress of Creative Activity and Brain Activity", Proceedings of the 8th IIAE International Conference on Industrial Application Engineering, pp. 21-25, 2020

(8) Sarnoff A. Mednick : "The associative basis of the creative process", Psychological Review, Vol. 69, N0. 3, pp. 220-232, 1962

(9) Valentina Quaresima, Silvia Bisconti and Marco Ferrari : "A brief review on the use of functional nearinfrared spectroscopy (fNIRS) for language imaging studies in human newborns and adults", Brain \& Language, Vol. 121, No. 2, pp. 79-89, 2012

(10) Peter H. Rudebeck and Erin L. Rich : “Orbitofrontal Cortex”, Current Biology, Vol. 28, No. 18, pp. R1083R1088, 2018

(11) Uma R. Mohan, Andrew J. Watrous, Jonathan F. Miller, Bradley C. Lega, Michael R. Sperling, Gregory A. Worrell, Robert E. Gross, Kareem A. Zaghloul, Barbara C. Jobst, Kathryn A. Davis, Sameer A. Sheth, Joel M. Stein, Sandhitsu R. Das, Richard Gorniak, Paul A. Wanda, Daniel S. Rizzuto, Michael J. Kahana and Joshua Jacobs : "The effects of direct brain stimulation in humans depend on frequency, amplitude, and whitematter proximity", Brain Stimulation, Vol. 13, No. 5, pp. 1183-1195, 2020

(12) Ying Zu Huang, Ming Kue Lu, Andrea Antal, Joseph Classen, Michael Nitsche, Ulf Ziemann, Michael Ridding, Masashi Hamada, Yoshikazu Ugawa, Shapour Jaberzadeh, Antonio Suppa, Walter Paulus and John Rothwell : "Plasticity induced by non-invasive transcranial brain stimulation: A position paper", Clinical Neurophysiology, Vol. 128, No. 12, pp. 23182329, 2017

(13) Vincent Ducoulombier, Roman Chiquet, Sahara Graf, Bernard Leroy, Guillaume Bouquet, Stéphane Verdun, Fabienne Martellier, Adeline Versavel, Alexandre Kone, Karine Lacroix, Didier Duthoit, Quentin Lenglet, Amelie Devaux, Robert Jeanson, Antoine Lefebvre, 
Benoit Coviaux, Gauthier Calais, Adeline Grimbert, Marie Ledein, Marie Moukagni, Tristan Pascart and Eric Houvenagel : "Usefulness of a Visual Analog Scale for Measuring Anxiety in Hospitalized Patients Experiencing Pain: A Multicenter Cross Sectional Study", Pain Management Nursing, Article in Press, 2020

(14) Satoshi Watanabe and Yuji Matsumoto : "An Analysis of Information Skills for Students Based on a Visual Analog Scale", Biomedical Fuzzy Systems Association, Vol. 13, No. 1, pp. 57-62, 2011 (in Japanese)

(15)David Attwell, Alastair M. Buchan, Serge Charpak, Martin Lauritzen, Brian A. MacVicar and Eric A. Newman : "Glial and neuronal control of brain blood flow”, Nature, Vol. 468, No. 7321, pp. 232-243, 2010

(16) Christoph Leithner, Georg Royl, Nikolas Offenhauser, Martina Füchtemeier, Matthias Kohl-Bareis, Arno Villringer, Ulrich Dirnagl and Ute Lindauer : "Pharmacological uncoupling of activation induced increases in CBF and CMRO2", Journal of Cerebral Blood Flow \& Metabolism, Vol. 30, No. 2, pp. 311-322, 2010

(17) Yihong Yang, Wolfgang Engelien, Hong Pan, Su Xu, David A. Silbersweig and Emily Stern : "A CBF-Based Event-Related Brain Activation Paradigm: Characterization of Impulse-Response Function and Comparison to BOLD”, NeuroImage, Vol. 12, No. 3, pp. 287-297, 2000

(18) Yoed N. Kenett, John D. Medaglia, Roger E. Beaty, Qunlin Chen, Richard F. Betzel, Sharon L. ThompsonSchill and Jiang Qiu : "Driving the brain towards creativity and intelligence: A network control theory analysis", Neuropsychologia, Vol. 118, No. 12, pp. 7990, 2018

(19) Paul A. Howard-Jones, Sarah-Jayne Blakemore, Elspeth A. Samuel, Ian R. Summers and Guy Claxton : "Semantic divergence and creative story generation: An fMRI investigation”, Cognitive Brain Research, Vol. 25, No. 1, pp. 240-250, 2005

(20) Akinori Hirano, Shuichi Yamamoto, Kazuki Yanagisawa and Hitoshi Tsunashima : "Evaluation of pleasant and unpleasant emotion evoked by visual stimuli using NIRS", Journal of the Human Interface Society, Vol. 17, No. 3, pp. 297-306 2015 (in Japanese) 